\title{
Effect of preconceptional orlistat treatment on in-vitro fertilization outcome in overweight/obese women: study protocol for a randomized controlled trial
}

\author{
Jing $\mathrm{Li}^{1 \dagger}$, Ze Wang ${ }^{1 \dagger}$, Daimin Wei ${ }^{1}$, Hong Liu', Jiangtao Zhang ${ }^{1}$, Jianfeng Wang ${ }^{1}$, Yuhua Shi ${ }^{* *}$ and Zi-Jiang Chen ${ }^{1,2}$
}

\begin{abstract}
Background: Obese women have fewer oocytes retrieved, an increased cancelation rate, a higher miscarriage rate, and a lower live birth rate after assisted reproductive technology (ART) treatment compared with women with normal weight. Weight loss before ART treatment can significantly improve pregnancy rates and/or live births. An orlistat plus diet intervention could promote weight loss, but there is no evidence from randomized clinical trials evaluating the effect of orlistat preconceptional treatment on pregnancy outcome in overweight and obese women.
\end{abstract}

Methods/design: We are conducting a multicenter, randomized placebo-controlled, double-blind clinical trial in overweight and obese women aged 20-40 years undergoing in-vitro fertilization and embryo transfer (IVF-ET) with or without intracytoplasmic sperm injection, to evaluate whether orlistat treatment for 1-3 months before IVF-ET can improve the live birth rate. The primary outcome is live birth.

Discussion: The results of this study will provide evidence for the effect of preconceptional orlistat treatment on IVF outcome in overweight/obese women.

Trial registration: Chinese Clinical Trial Registry, ChiCTR-IPR-17011629. Registered on 11 June 2017.

Keywords: Orlistat, Overweight, Obese, Randomized controlled trial, Live birth

\section{Background}

The prevalence of obesity is increasing worldwide, and it causes not only an increased risk of metabolic syndrome, type 2 diabetes, and cardiovascular disease, but also a decline in fertility and an increased risk of infertility [1]. Obese women have a higher dose of gonadotrophin, more days of stimulation, fewer oocytes [2, 3], an increased cancelation rate, a higher miscarriage rate [4], and a lower live birth rate $[5,6]$ compared with women with normal weight. According to a systematic review by Maheshwari et al. [7], women with $\mathrm{BMI} \geq 25 \mathrm{~kg} / \mathrm{m}^{2}$ have

\footnotetext{
* Correspondence: shiyuhua2003@126.com

${ }^{\dagger}$ Jing Li and Ze Wang contributed equally to this work.

${ }^{1}$ Center for Reproductive Medicine, Shandong Provincial Hospital Affiliated to Shandong University, Key Laboratory of Reproductive Endocrinology, Shandong University, Ministry of Education, and National Research Center for Assisted Reproductive Technology and Reproductive Genetics, Jinan, China Full list of author information is available at the end of the article
}

a lower chance of pregnancy and an increased miscarriage rate following IVF. Another meta-analysis showed similar results that women with $\mathrm{BMI} \geq 25 \mathrm{~kg} / \mathrm{m}^{2}$ had significantly lower live birth rates compared to those with $\mathrm{BMI}<25 \mathrm{~kg} / \mathrm{m}^{2}$ following IVF/ICSI treatment [8]. The risk for preeclampsia and gestational diabetes was doubled in obese pregnant women [9], and maternal obesity was associated with an increased risk of many structural anomalies $[10,11]$. Short-term weight loss was associated with higher metaphase II (MII) oocyte yield, but unrelated to clinical pregnancy or live birth rates [12]. Another study reported that a "meaningful" weight loss of $10 \%$ could significantly improve live birth rates (71\% vs 37\%) [13]. A systematic review showed that women with weight loss before ART treatment had significantly improved pregnancy rates and/or live births [14]. 
A high-caloric diet is the main cause of obesity, and a higher fat intake is associated with dyslipidemia and insulin resistance. The common strategy to lose weight includes modification of unhealthy dietary habits, more physical activity, pharmacological management, and bariatric surgery. But poor patient compliance with lifestyle modification is known because it needs a long time to take effect. Therefore, it is reasonable to focus on the consumption of fat to lose weight.

Orlistat prevents the absorption of triglyceride from the human diet by irreversibly combining with gastric and pancreatic lipase, and it is reported that an orlistat plus diet intervention could decrease weight, cholesterol, and insulin levels $[15,16]$. But there is no report about the effect of orlistat before ART treatment on pregnancy outcome in overweight and obese women.

\section{Methods/design}

\section{Design and setting}

This study is a multicenter, randomized placebo-controlled, double-blind clinical trial to evaluate whether orlistat treatment for 1-3 months before IVF-ET can improve the live birth rate. Patients are recruited from 19 hospitals in China. In this study, overweight is defined as $\mathrm{BMI} \geq 25 \mathrm{~kg} / \mathrm{m}^{2}$ and obesity as BMI $\geq 28 \mathrm{~kg} / \mathrm{m}^{2}$ according to Chinese criteria.

\section{Inclusion criteria}

The inclusion criteria are as follows:

1. Women aged $\geq 20$ and $\leq 40$ years.

2. Women with $\mathrm{BMI} \geq 25 \mathrm{~kg} / \mathrm{m}^{2}$.

3. Women who are undergoing IVF or intracytoplasmic sperm injection (ICSI).

\section{Exclusion criteria}

The exclusion criteria are as follows:

1. Women who had no clinical pregnancy after three or more cycles of IVF/ICSI.

2. Women who have been diagnosed with congenital or acquired uterine abnormalities by ultrasound or with severe intrauterine adhesion by hysteroscope.

3. Women with abnormal liver or renal function.

4. Women with an allergy to orlistat.

5. Women with a diagnosis of malabsorption syndrome or cholestasis.

6. Women with organic obesity; for example, hypothyroidism.

\section{Sample size}

Based on the retrospective data from our IVF clinic, the live birth rate of obese or overweight women is about $35 \%$. It is assumed that an improvement of $10 \%$ in the live birth rate will be clinically significant. We aim to test a difference of $10 \%$ in the live birth rate between treatment groups (45\% in the orlistat group) at a significance level of 0.05 with a statistical power of $80 \%$. The minimal sample size calculated is 752 , and 836 participants will be enrolled in consideration of a dropout rate of $10 \%$.

\section{Screening}

At the screening visit, patients who have been using orlistat or other weight-reducing aid/drugs will be excluded, and the couples sign the written informed consent after safety assessment, which includes fasting blood glucose, insulin, triglyceride (TG), total cholesterol (TC), high density lipoprotein (HDL), low density lipoprotein (LDL), lipoprotein a (Lp (a)), liver function, renal function, complete blood count (CBC), coagulation test, hepatitis $\mathrm{B}$ virus (HBV), hepatitis $\mathrm{C}$ virus ( $\mathrm{HCV})$, human immunodeficiency virus (HIV), syphilis, and routine urine. The standardized case report forms are completed to collect current medication status and previous medical history. A physical examination (height, body weight, waistline, hipline, blood pressure) and transvaginal ultrasound scan are performed. The basal sex hormones are tested in the local laboratories of the study sites. A diagnostic hysteroscopy is performed in patients who are suspected to have an abnormal uterine cavity after ultrasound scan. All participants are prescribed multivitamins and advised to have a healthy lifestyle, more physical activity, and a reduced-calorie, low-fat diet, and to avoid gaining weight rapidly during pregnancy. A schedule of enrollment, interventions, and assessment is provided in the Standard Protocol Items: Recommendations for Interventional Trials (SPIRIT) figure (Fig. 1). The flow chart of this study is shown in Fig. 2. The SPIRIT checklist is presented in Additional file 1.

\section{Randomization and blinding}

A total of 836 patients will be enrolled, and a computergenerated random list generated by SPSS 16.0 (SPSS Inc., Chicago, IL, USA) will be used for randomization. The packaging is marked according to the random number list; the packaging and tablets of orlistat (Huadong Pharmaceutical Co., Ltd) and the placebo have the same appearance, which cannot be distinguished. The placebo is manufactured by Huadong Pharmaceutical Co., Ltd, and, in addition to the active ingredients, the rest of the excipient and the appearance and odor are exactly the same as orlistat. The random number list is kept strictly confidential by the Data Coordination Committee (DCC) staff, and the researchers who are in charge of the enrollment have no access to the list. Study personnel are blinded to upcoming treatment group allocation. Participants will receive the medication (orlistat or placebo with a 1:1 ratio). The appearance of orlistat and placebo is identical in packaging, and the participants and all research 


\begin{tabular}{|c|c|c|c|c|c|c|c|c|c|c|}
\hline & \multicolumn{10}{|c|}{ STUDY PERIOD } \\
\hline & \multirow{2}{*}{$\begin{array}{c}\text { Enrolment } \\
\text { to } \\
\end{array}$} & \multicolumn{9}{|c|}{ Post-allocation } \\
\hline TIMEPOINT & & t1 & t2 & t3 & t4 & t5 & t6 & t7 & t8 & T9 \\
\hline \multicolumn{11}{|l|}{ ENROLMENT: } \\
\hline Eligibility screen & $x$ & & & & & & & & & \\
\hline Safety assessment & $x$ & & & & & & & & & \\
\hline Informed consent & $x$ & & & & & & & & & \\
\hline Allocation & $x$ & & & & & & & & & \\
\hline \multicolumn{11}{|l|}{ INTERVENTIONS: } \\
\hline orlistat group & $x$ & & & & & & & & & \\
\hline [placebo group] & $x$ & & & & & & & & & \\
\hline \multicolumn{11}{|l|}{ ASSESSMENTS: } \\
\hline Physical examination & $x$ & $x$ & $\mathrm{x}$ & & & & & & & \\
\hline Ultrasonography & $x$ & & & & $x$ & $x$ & & & & \\
\hline Pregnancy test & & & & $\mathrm{x}$ & & & & & & \\
\hline \multirow{2}{*}{ Telephone follow-up } & & & & & & & $x$ & $x$ & & \\
\hline & & & & & & & & & $x$ & $x$ \\
\hline
\end{tabular}

Fig. 1 SPIRIT diagram for schedule of enrollment, interventions, and assessments. Safety assessment includes fasting blood glucose, insulin, TG, TC, HDL, LDL, Lp (a), liver function, renal function, CBC, coagulation test, HBV, HCV, HIV, syphilis, and routine urine. t1, 1-3 months after allocation; t2, day of $E T ; t 3,2$ weeks after $E T ; t 4,5$ weeks after $E T ; t 5,10$ weeks after $E T ; t 6,28$ weeks of gestation; $t 7,37$ weeks of gestation; $t 8$, delivery; $t 9$, 6 weeks after delivery

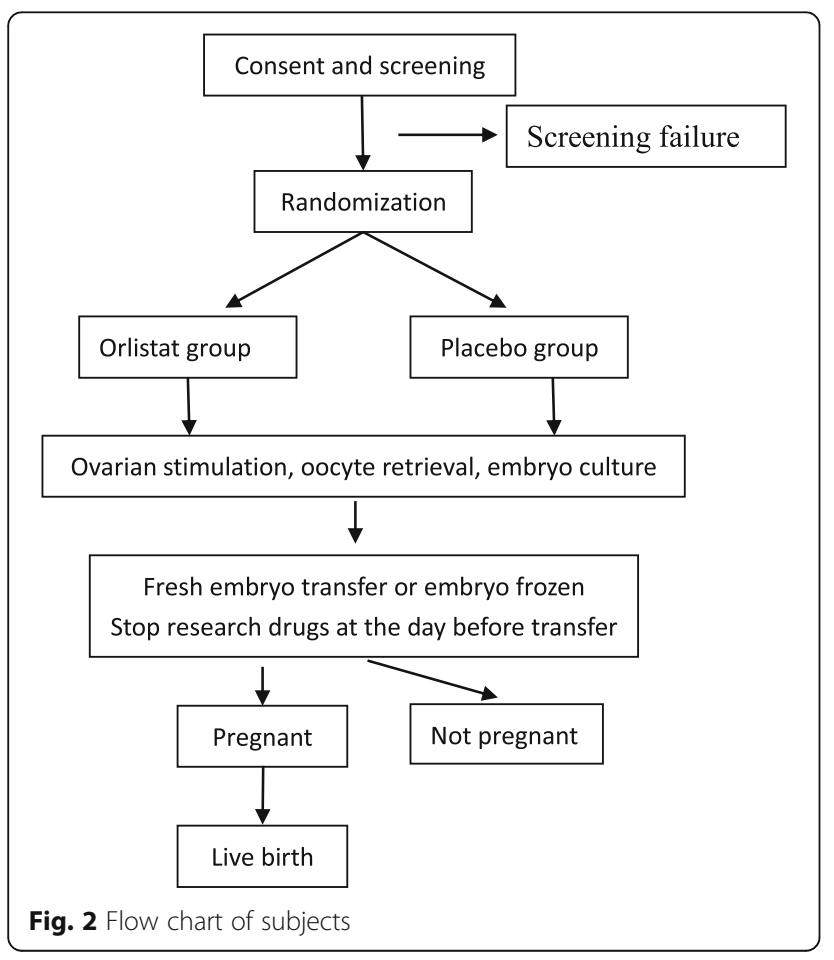

staff do not know the allocation until the end of the study. The quality of the placebo, such as contents and bacteria contaminations, was controlled rigorously according to the GMP standard.

\section{Interventions}

Participants are randomized to receive either orlistat $120 \mathrm{mg}$ p.o. three times daily (intervention) or placebo $120 \mathrm{mg}$ p.o. three times daily after signing consent at the screening visit. Both groups of participants will take orlistat or placebo for 1-3 months before ovarian stimulation and to the day of fresh embryo transfer.

The protocol of ovarian stimulation is decided by the physicians according to the patients' characteristics. Oocyte retrieval is performed 34-36 h after human chorionic gonadotrophin (hCG) injection by experienced physicians, and oocytes are inseminated by IVF or ICSI according to the quality of sperm. On day 3 or 5 of the embryo culture, the quality of embryos is assessed by experienced embryologists, and the morphological criteria are selected by the local laboratory. No more than three embryos on day 3 or 5 will be transferred, and the rest of the embryos will be frozen according to local experience. The protocol of luteal phase support and the day of starting will be determined by the local center. The serum hCG test is performed after 2 weeks of transfer, 
and if the test is positive, follow-up will be continued to the end of pregnancy or delivery.

\section{Outcome and outcome assessments}

The primary outcome is live birth after first fresh embryo transfer. The secondary outcomes include changes of body weight, moderate and severe ovarian hyperstimulation syndrome (OHSS), dose of gonadotrophin during ovarian stimulation, numbers of oocytes, embryos of high quality, conception, clinical pregnancy, ongoing pregnancy, miscarriage, pregnancy and perinatal complication, birth weight, neonatal complication, and other adverse events.

Conception is defined with the result of serum $\beta$-hCG $\geq 10 \mathrm{mIU} / \mathrm{ml}$ measured 2 weeks after embryo transfer.

Clinical pregnancy is defined as detection of a gestational sac in the uterine cavity by a transvaginal ultrasound scan 20 days after conception confirmation.

An ectopic pregnancy is defined as a complication of pregnancy in which the embryo implants at any site other than the endometrial lining of the uterine cavity.

Ongoing pregnancy is defined as detection of a viable fetus with fetal heartbeat at 11-12 weeks of gestation.

Miscarriage is defined as pregnancies that eventuate in a spontaneous abortion before 20 weeks of gestation.

A live birth is defined as the delivery of any number of newborns at $\geq 28$ weeks of gestation with heartbeat and breath.

\section{Safety assessment}

Adverse events (AEs) are any untoward medical occurrences associated with the subject's participation in the research, which do not necessarily have a causal relationship with the study intervention. Serious adverse events (SAEs) are events that occur during the subject's participation in research that meet any of the following criteria: death, life-threatening, severe or persistent disability, requiring inpatient hospitalization or prolongation of existing hospitalization, neonatal death up to 6 weeks after delivery, congenital anomaly or birth defect, or any events deemed serious by the local principal investigator.

Participants will be questioned to report all AEs and unintended concomitants medications at each visit, and all reported AEs will be assessed and recorded. All abnormal changes of blood tests will be evaluated by the investigators. SAEs will be reported in 5 days; SAEs which are unintended and possibly associated with interventions will be reported within $24 \mathrm{~h}$.

\section{Data collection and management}

To ensure the accuracy of outcome assessments and data collection, all of the physicians, nurses, and research assistants will attend a training workshop before the start of the trial. All attenders will be provided with a protocol and standard operation procedures, and will discuss the topics they may feel confused about until everyone is totally clear about the procedures. The DCC is responsible for the monitoring tasks of the trial. Personnel of Shandong University will regularly check the data from different sites, determine the issues that affect the quality and speed of research, and take corresponding measures.

After screening and signing of informed consent, the current medication status, previous medical history, height, body weight, waistline, hipline, blood pressure, transvaginal ultrasound scan, and basal sex hormones will be recorded.

OHSS, dose of gonadotrophin, and information on oocytes and embryos will be assessed from the start of ovarian stimulation to the day of the pregnancy test.

Serum $\beta$-hCG will be measured 2 weeks after embryo transfer. If the serum $\beta$-hCG test is positive, a transvaginal ultrasound scan will be performed 20 days later to detect the gestational sac.

During pregnancy beyond 12 weeks, participants are contacted by telephone call at 28 and 32 weeks to inquire about pregnancy complications, AEs, and combination therapy.

At delivery, obstetrical and perinatal complications and neonatal information are obtained with reference to obstetric medical records and neonatal medical records.

At 6 weeks after delivery, postpartum complications and neonatal complications are followed up by telephone call.

Data are collected and recorded on a standard case report form, and when the visit is completed in every site, all of the recorded data will be entered into the web-based data system by the double-entry method.

Any participant may quit the trial at any time for any reason. If any patients want to quit, clinicians will ask whether they agree to finish the follow-up according to the trial schedule. All patients who quit and are lost to follow-up will be recorded.

\section{Data analysis plan}

The data will be analyzed by SPSS 16.0 (SPSS Inc., Chicago, IL, USA). The analysis will be conducted using intention-to-treat principles. The primary outcome, the live birth rate, will be compared between two groups using the Pearson chi-square test; secondary outcome parameters, such as the pregnancy rate, OHSS rate, and other rates, will be analyzed using the Pearson chi-square test. A per-protocol analysis will be performed according to the actual participants completing the entire trial. Continuous data are expressed as mean \pm standard deviation, with Student's test for data with normal distribution and Wilcoxon rank-sum test for nonnormal distribution. Categorical data are represented as frequency and percentage 
with chi-square analysis or Fisher's exact test for expected frequencies less than $5 . P<0.05$ will be considered significant.

The reasons for missing data will be recorded, and according to these reasons, four approaches may be considered: complete-case analysis, single imputation methods, estimating-equation methods, and methods based on a statistical model.

\section{Discussion}

This is a trial evaluating the effect of preconceptional orlistat treatment on pregnancy outcome in overweight and obese women aged 20-40 years after IVF. We plan to enroll 836 subjects from 19 hospitals in China. The enrollment began in July 2017. At the time of manuscript preparation, more than 350 subjects have been enrolled. The result of this multicenter randomized trial will provide valid evidence for the effect of orlistat pretreatment on IVF pregnancy outcome in overweight and obese women. We speculate that taking orlistat before ART treatment may improve the pregnancy outcome in overweight and obese women.

\section{Trial status}

The enrollment is ongoing at the time of manuscript submission.

\section{Additional file}

Additional file 1: SPIRIT checklist. (DOC $125 \mathrm{~kb}$ )

\begin{abstract}
Abbreviations
AE: Adverse event; ART: Assisted reproductive technology; BMI: Body mass index; CBC: Complete blood count; DCC: Data Coordination Committee; hCG: Human chorionic gonadotrophin; HDL: High density lipoprotein; ICSI: Intracytoplasmic sperm injection; IVF: In-vitro fertilization; LDL: Low density lipoprotein; Lp (a): Lipoprotein a; OHSS: Ovarian hyperstimulation syndrome; SAE: Serious adverse event; TC: Total cholesterol; TG: Triglyceride
\end{abstract}

\section{Acknowledgements}

The authors thank all of the patients for their voluntary participation in this trial and the physicians at all study sites for referring subjects.

\section{Funding}

This research did not receive any specific grant from funding agencies in the public, commercial, or not-for-profit sectors.

\section{Availability of data and materials}

The datasets generated during the current study are available from the corresponding author on reasonable request.

\section{Authors' contributions}

$J \mathrm{~L}, \mathrm{ZW}$, and DW were involved in the study concept and design and in drafting of the manuscript. YS contributed to the study design and critical revision of the manuscript. $\mathrm{HL}, \mathrm{JZ}$, and JW contributed to the study design and revision of the manuscript. YS and Z-JC were involved in the study concept and design and in revision of the manuscript. All authors read and approved the final manuscript.

\section{Ethics approval and consent to participate}

The protocol has been approved by the institutional review board (IRB) at the Reproductive Medicine Center affiliated to Shandong University (2017 IRB approval No. 23). Informed consent will be obtained from all participants prior to enrollment.

\section{Consent for publication}

Not applicable.

\section{Competing interests}

The authors declare that they have no competing interests.

\section{Publisher's Note}

Springer Nature remains neutral with regard to jurisdictional claims in published maps and institutional affiliations.

\section{Author details}

${ }^{1}$ Center for Reproductive Medicine, Shandong Provincial Hospital Affiliated to Shandong University, Key Laboratory of Reproductive Endocrinology, Shandong University, Ministry of Education, and National Research Center for Assisted Reproductive Technology and Reproductive Genetics, Jinan, China.

${ }^{2}$ Center for Reproductive Medicine, Ren Ji Hospital, School of Medicine, Shanghai Jiao Tong University, Shanghai Key Laboratory for Assisted Reproduction and Reproductive Genetics, Shanghai, China.

Received: 21 December 2017 Accepted: 2 July 2018

Published online: 18 July 2018

References

1. Talmor A, Dunphy B. Female obesity and infertility. Best Pract Res Clin Obstet Gynaecol. 2015;29:498-506.

2. Zhang D, Zhu Y, Gao H, Zhou B, Zhang R, Wang T, Ding G, Qu F, Huang H, Lu X. Overweight and obesity negatively affect the outcomes of ovarian stimulation and in vitro fertilisation: a cohort study of 2628 Chinese women. Gynecol Endocrinol. 2010;26:325-32.

3. Setti AS, Braga DP, Figueira Rde C, Vingris L, laconelli A, Borges E Jr. Body mass index is negatively correlated with the response to controlled ovarian stimulation but does not influence oocyte morphology in ICSI cycles. Eur J Obstet Gynecol Reprod Biol. 2012;163:175-9.

4. Caillon H, Freour T, Bach-Ngohou K, Colombel A, Denis MG, Barriere P, Masson D. Effects of female increased body mass index on in vitro fertilization cycles outcome. Obes Res Clin Pract. 2015:9:382-8.

5. Fedorcsak P, Dale PO, Storeng R, Ertzeid G, Bjercke S, Oldereid N, Omland AK, Abyholm T, Tanbo T. Impact of overweight and underweight on assisted reproduction treatment. Hum Reprod. 2004;19:2523-8.

6. Moragianni VA, Jones SM, Ryley DA. The effect of body mass index on the outcomes of first assisted reproductive technology cycles. Fertil Steril. 2012; 98:102-8.

7. Maheshwari A, Stofberg L, Bhattacharya S. Effect of overweight and obesity on assisted reproductive technology — a systematic review. Hum Reprod Update. 2007;13:433-44.

8. Rittenberg V, Seshadri S, Sunkara SK, Sobaleva S, Oteng-Ntim E, El-Toukhy T. Effect of body mass index on IVF treatment outcome: an updated systematic review and meta-analysis. Reprod BioMed Online. 2011;23:421-39.

9. Dean SV, Lassi ZS, Imam AM, Bhutta ZA. Preconception care: nutritional risks and interventions. Reprod Health. 2014;11(Suppl 3):S3.

10. Stothard KJ, Tennant PW, Bell R, Rankin J. Maternal overweight and obesity and the risk of congenital anomalies: a systematic review and meta-analysis. Jama. 2009:301:636-50.

11. Rasmussen SA, Chu SY, Kim SY, Schmid CH, Lau J. Maternal obesity and risk of neural tube defects: a metaanalysis. Am J Obstet Gynecol. 2008;198:611-9.

12. Chavarro JE, Ehrlich S, Colaci DS, Wright DL, Toth TL, Petrozza JC, Hauser R. Body mass index and short-term weight change in relation to treatment outcomes in women undergoing assisted reproduction. Fertil Steril. 2012;98: $109-16$

13. Kort JD, Winget C, Kim SH, Lathi RB. A retrospective cohort study to evaluate the impact of meaningful weight loss on fertility outcomes in an overweight population with infertility. Fertil Steril. 2014;101:1400-3.

14. Sim KA, Partridge SR, Sainsbury A. Does weight loss in overweight or obese women improve fertility treatment outcomes? A systematic review. Obes Rev. 2014;15:839-50. 
15. Sjostrom L, Rissanen A, Andersen T, Boldrin M, Golay A, Koppeschaar HP, Krempf M. Randomised placebo-controlled trial of orlistat for weight loss and prevention of weight regain in obese patients. European Multicentre Orlistat Study Group. Lancet. 1998;352:167-72.

16. Davidson MH, Hauptman J, DiGirolamo M, Foreyt JP, Halsted CH, Heber D, Heimburger DC, Lucas CP, Robbins DC, Chung J, Heymsfield SB. Weight control and risk factor reduction in obese subjects treated for 2 years with orlistat: a randomized controlled trial. JAMA. 1999;281:235-42.

Ready to submit your research? Choose BMC and benefit from:

- fast, convenient online submission

- thorough peer review by experienced researchers in your field

- rapid publication on acceptance

- support for research data, including large and complex data types

- gold Open Access which fosters wider collaboration and increased citations

- maximum visibility for your research: over $100 \mathrm{M}$ website views per year

At BMC, research is always in progress.

Learn more biomedcentral.com/submissions 